\title{
Krastev, Ivan/Holmes, Stephen: Das Licht, das erlosch. Eine Abrechnung, übers. v. Karin Schuler, 368 S., Ullstein, Berlin 2019.
}

\author{
Jens Hacke \\ Online publiziert: 27. Juli 2020 \\ (C) Der/die Autor(en) 2020
}

Dem ebenso anregenden wie streitbaren Großessay von Ivan Krastev und Stephen Holmes im Rahmen einer knappen Besprechung gerecht zu werden, ist ein Ding der Unmöglichkeit. Man kommt nicht umhin, die Fülle an originellen Perspektiven, den Gedankenreichtum und das kompositorische Geschick zu loben. Zugleich sind allerdings begriffliche Unschärfen und die Vagheit kollektivpsychologischer Erklärungen zu markieren. Das Autorengespann zeichnet ein düsteres Szenario vom drohenden Scheitern der liberalen Demokratie. Auch hier muss der beinahe totzitierte Francis Fukuyama wieder herhalten: als Beispiel für einen Triumphalismus, der den Westen nach einem unverschuldet und überraschend eingeheimsten Sieg über den implodierenden Systemgegner hat so träge werden lassen, dass er zum Opfer seines Erfolges wird. Etwas vereinfachend monieren die Verfasser, dass ,,der Liberalismus zugunsten einer Hegemonie die Alternativen aufgegeben“ (S. 15) und es sich am „Ende der Geschichte“ zu bequem gemacht habe.

Im Zentrum steht die Frage, wie es eigentlich zur Krise der Demokratie insgesamt und zur Konjunktur antiliberaler und autoritärer Bewegungen kommen konnte. Dabei rücken die Verfasser vor allem die Dimension der internationalen Ordnung und der außenpolitischen Interessen sowie die mentale Disposition der Transformationsgesellschaften im ehemaligen Ostblock ins Blickfeld. Die Spezifik der Revolutionen in Ost- und Mitteleuropa lokalisieren sie in ihrem außengeleiteten Charakter. Die freiheitlichen Ideale der Bürgerrechtsbewegungen und Konsumerwartungen der breiten Massen orientierten sich am westlichen Vorbild, aber die „,nachholenden Revolutionen“ (Jürgen Habermas) von 1989 brachten keine eigenen neuen Ideen hervor.

Im Thema der ,Nachahmung' offenbart sich bereits eine Problematik, die das Buch durchzieht. Im Modus der Imitation eifern politische Gemeinschaften vermeintlich erfolgreicheren Vorbildern nach. Zugrunde gelegt wird gewissermaßen eine Vorstellung von nationalkultureller Authentizität, deren Ideal eigentlich nur verfehlt werden kann. Krisen werden dann zur Abfolge von Entfremdungserfah-

\footnotetext{
J. Hacke $(\bowtie)$

Humboldt-Universität zu Berlin, Berlin, Deutschland

E-Mail: jens.hacke@gmx.net

Universität der Bundeswehr München, München, Deutschland
} 
rungen - ob in sowjetisch aufgezwungenen Volksrepubliken oder in der eilfertigen Übernahme des westlichen Systems ohne die Internalisierung demokratischer Werte, zivilgesellschaftlicher Praxis oder marktwirtschaftlicher Ordnung. Natürlich geraten solche kollektivpsychologischen Deutungen etwas holzschnittartig. Dennoch hilft das grobe Raster der Autoren manche Entwicklungen besser zu erkennen. Es war das Eigentümliche der osteuropäischen Revolutionen, dass viele Sieger das jeweilige Land verließen, also eine vorher kaum je erlebte Abwanderung ungeduldiger Eliten einsetzte, während die Verlierer blieben. Migration wurde als Kapitulation erlebt, und so schien es kompensatorisch nötig, die Demokratie als ein nationales Projekt kultureller Homogenität zu begründen. Hinzu kam die in ungeheurer Geschwindigkeit forcierte EU-Erweiterung, deren Vorgaben einem Oktroi des Westens glichen. Das Autorengespann zeichnet ein Bild enttäuschter Erwartungen und sieht die Ursprünge für den „Aufstieg des autoritären Chauvinismus und der Fremdenfeindlichkeit [...] in der politischen Psychologie, nicht in der politischen Theorie“" (S. 111). Sie melden damit Zweifel an den ökonomischen Erklärungen des Populismus an und wollen zeigen, wie der Osten nunmehr nach dem Westen greift.

Die Erklärungen, die eine Traumatologie der Transition hervorbringen, wirken bisweilen sehr suggestiv. Aber insbesondere die Analyse der russischen Mimikry, die Krastev und Holmes als Simulation einer Demokratisierung und strategische Kopie westlicher Rhetoriken begreifen, kann ein hohes Maß an Plausibilität entfalten. Auf den Pfaden von Masha Gessen und Timothy Snyder arbeiten die Autoren sehr lesenswert heraus, wie geschickt und suggestiv Vladimir Putin außenpolitisches Handeln mit dem Einsatz für demokratische Selbstbestimmung und für Menschenrechte verbrämt - ein Missbrauch von Werten, den bereits die USA eingeleitet haben und der die moralische Integrität der liberalen Demokratie untergraben hat. Mit genauem Blick analysieren die Verfasser die manipulative Seite demokratischen Regierens, die seit den Demagogen der Antike thematisiert wird und noch die sogenannte „realistische Demokratietheorie“ im Anschluss an Joseph Schumpeter bestimmt.

Wenig erfährt der Leser über die Lage der parlamentarischen Demokratie oder über die innere Konstitution demokratischer Gesellschaften. Auch das Phänomen Donald Trump ist als Modell umgedrehter Imitation schwer zu verstehen, wenn man die intrinsischen Probleme der politischen Kultur in den USA ignoriert. Der Westen bleibt in der vorliegenden Lesart ohne eigene Agenda dem weltpolitischen Ringen mit einer autokratischen Internationale ausgeliefert, deren Ziele entweder rein destruktiv und antiliberal sind - wie im Falle Putins - oder deren Politik einem kühlen ideologisch enthaltsamen ökonomischen Interesse und der Machtakkumulation dient, wie das Beispiel des „,chinesischen Neomerkantilismus“ zeigt (S. 289). Zum strategischen Nachteil in dieser Auseinandersetzung geraten schließlich die normativen Eckpfeiler der Demokratie: Die offene Gesellschaft wird zum Einfallstor für Migration und Manipulation; Transparenz wird zur Schwäche, weil der Gegner die Spielzüge der Demokratien wie in einem offenen Buch lesen kann; das Wahrheitspostulat verschafft den Lügen der Gegner immer einen Vorsprung durch Ablenkung und falsche Fährten, die mühsam entschlüsselt werden müssen, ohne dass dies Konsequenzen nach sich zöge.

Überrascht registriert die Leserin inmitten dieser Lust am Untergang, dass Krastev und Holmes am Ende dafür plädieren, ,unsere Rückkehr in eine Welt ständig 
miteinander rangelnder politischer Alternativen zu feiern“, um einen ,geläuterten Liberalismus“ wieder aufzurichten (S. 304). Was allerdings unter Liberalismus zu verstehen ist - und wie die liberale Demokratie auf innere und äußere Herausforderungen reagieren soll, bleibt nach der Lektüre unklar. Für die Autoren ist der liberale Nationalstaat weltweit ,die effektivste Menschenrechtsorganisation“ (S. 89), und eigentlich kehren sie damit zum vielgescholtenen Fukuyama zurück - nur dass sie den Abschied vom Universalismus mittlerweile für unvermeidlich halten.

Funding Open Access funding provided by Projekt DEAL.

Open Access Dieser Artikel wird unter der Creative Commons Namensnennung 4.0 International Lizenz veröffentlicht, welche die Nutzung, Vervielfältigung, Bearbeitung, Verbreitung und Wiedergabe in jeglichem Medium und Format erlaubt, sofern Sie den/die ursprünglichen Autor(en) und die Quelle ordnungsgemäß nennen, einen Link zur Creative Commons Lizenz beifügen und angeben, ob Änderungen vorgenommen wurden.

Die in diesem Artikel enthaltenen Bilder und sonstiges Drittmaterial unterliegen ebenfalls der genannten Creative Commons Lizenz, sofern sich aus der Abbildungslegende nichts anderes ergibt. Sofern das betreffende Material nicht unter der genannten Creative Commons Lizenz steht und die betreffende Handlung nicht nach gesetzlichen Vorschriften erlaubt ist, ist für die oben aufgeführten Weiterverwendungen des Materials die Einwilligung des jeweiligen Rechteinhabers einzuholen.

Weitere Details zur Lizenz entnehmen Sie bitte der Lizenzinformation auf http://creativecommons.org/ licenses/by/4.0/deed.de. 